\title{
Remote Health Monitoring System in a Rural Population: Challenges and Opportunities
}

\author{
Jacey-Lynn Minoi IEEE Member and Alvin W Yeo IEEE Member
}

\begin{abstract}
This paper discusses remote health monitoring as a potential application field in telecentres at rural areas. We present the challenges faced and opportunities with emphasis on patients with hypertension using a remote health monitoring system. We will also discuss the potential of deploying mHealth applications as a value added to the telecentres. The remote blood pressure health monitoring system will read, store and send data over wireless network to a remote server. Medical doctors can view the data on a regular basis remotely from a website. This community-based participatory research study carried out BP monitoring on residents from a rural village in Sarawak, Borneo and did follow-up assessments on the available health care for them. Structured quantitative and qualitative research tools were carried out on a wide range of clients - the patients, telecentre managers and medical doctors. Structured and semi-structured data collection techniques, such as questionnaires, group discussions and interviews were gathered. Observation of the whole process of interaction between the patients with the managers was also captured. The aim of the project is to determine the degree to which remote health monitoring interventions can be integrated to telecentres in rural areas to increase awareness in healthy living and wellness.
\end{abstract}

\section{INTRODUCTION}

Primary hypertension is the one of most critical chronic illnesses affecting Malaysians over the past decades. It is known as a 'silent killer' as most hypertensive patients are asymptomatic and unaware of their clinical conditions with risk factor associated with cardiovascular, cerebrovascular and renal diseases $[1,2]$. It is also well known that earlier intervention for hypertension to achieve a more normal blood pressure carries a favourable outcome, but motivation and compliance are always a challenge [3, 4]. Limited systematic reviews have shown that structured telemonitoring may have positive impacts on the management of chronic illness, such as chronic heart failure and asthma $[5,6]$.

Blood pressure (BP) monitoring is vital for blood pressure management. Traditionally, BP of a patient is taken by a physician or a nurse in an office based medical care setting, such as in a clinic or a hospital. In this study, we proposed non-medical personnel to take BP measurement of patients at their homes or at a designated telecentre in the

Research supported by ISITI and ERICSSON fund under grant no. SHSB/B-AM2011/54

J. -L. Minoi is with the Faculty of Computer Science and Information Technology, Universiti Malaysia Sarawak, Malaysia (e-mail: jacey@fit.unimas.my).

Prof. A. W. Yeo is the Director of the Institute of Social Informatics and Technological Innovation - Rural Informatics, Malaysia (e-mail: alvin@isiti.unimas.my). village. The advantage of home BP measurement could remove 'white coat hypertension effect' - a phenomenon known as falsely high BP readings related to patients' reaction to clinical procedures in a typical clinical setting [4].

There is a high aging population living in a remote and rural village in Sarawak, Borneo, who are unable to visit hospitals in the city or clinics for regular check-ups. Given the high prevalence of hypertension in Malaysia, the need for a remote health monitoring system will be examined based on the initial consideration of the limited health care delivery to these remote and rural areas surrounded by dense jungle on mountainous terraces and limited public transportation available. We have conducted a participatory study at Serasot Village located in Bau district, which is about 50 kilometres away from the nearest city centre. There exist a telecentre in the village that is erected by the Malaysian Communication and Multimedia Commission (MCMC) to house computer equipment with an initial aim to provide some form of ICT services for social and economic development. The telecentres are as diverse as the communities they serve.

The aim of our work is to collaborate with MCMC and the nearby clinic to deploy a remote health monitoring system for the residents. With the increasing demand for cost-effective health care solutions, the use of ICT to enable long-distance health care may be the only economically viable way to make health care resources available and putting it in a telecentre is practical also for providers to deliver throughout the community. Our participatory study will capture behaviours, interactions and communications between patients and clinicians to be analysed for long-term collaboration health management.

\section{REMOte HeALth Monitoring}

The technological advances in mobile Health (mHealth) applications have been brought about by the availability of valid and easy-to-use automated ambulatory monitoring devices, such as blood pressure monitor, oximeter, glucometer, and weighting scale. The mHealth system we deployed to the community allows the measurements to be automatically transmitted via wireless $3 \mathrm{G}$ network or WIFI to a remote server whereby medical doctors can view the data on a regular basis remotely from a website through the mHealth application.

We used a commercialised Ericsson Mobile Health (EMH) [7] system that comes with several components: Patient Units (several medical sensors embedded with Bluetooth technology for blood pressure, pulse rate, body 